Homology, Homotopy and Applications, vol.16(1), 2014, pp.179-198

\title{
VERSAL DEFORMATION THEORY OF ALGEBRAS OVER A QUADRATIC OPERAD
}

\author{
ALICE FIALOWSKI, GOUTAM MUKHERJEE AND ANITA NAOLEKAR
}

(communicated by Claude Cibils)

\begin{abstract}
We develop deformation theory of algebras over quadratic operads where the parameter space is a commutative local algebra. We also give a construction of a distinguised deformation of an algebra over a quadratic operad with a complete local algebra as its base - the so-called versal deformation - which induces all other deformations of the given algebra.
\end{abstract}

In memory of our good friend Jean-Louis Loday

\section{Introduction}

Formal one-parameter deformation theory for algebras was originally introduced for associative algebras by M. Gerstenhaber in the 1960s (see [7, 8]). Since then it has been applied to many other algebraic categories. Most of these cases turned out to be algebras over a suitable quadratic operad. Gerstenhaber's theory was generalized to an algebra over a binary quadratic operad by D. Balavoine in 1997 (see [1]) and further developed by M. Kontsevich and Y. Soibelman (see [12]). In [13], J.L. Loday and B. Vallette introduced a deformation complex for any $\mathcal{P}$-algebra $A$ over a quadratic operad $\mathcal{P}$. It turns out that this deformation complex is the resulting $\mathrm{dg}$ Lie algebra obtained from convolution dg Lie algebra $\left.\mathfrak{g}_{\mathcal{P}, A}=\left(\operatorname{Hom}_{\mathbb{S}}\left(\mathcal{P}^{\mathrm{i}}, \operatorname{End}_{A}\right)\right),[],\right)$, where $\mathcal{P}^{\mathrm{i}}$ is the Koszul dual co-operad of $\mathcal{P}$, twisted by the algebra structure.

Classical deformation theory is not general enough to describe all nonequivalent deformations of a given object. To take care of this, one needs to enlarge the base of deformations from a one parameter power series ring to a local commutative algebra, or more generally, to a complete local algebra. It is known that under certain cohomological restrictions there exists a "characteristic" versal deformation of a type of algebras, with complete local algebra base, that induces all nonequivalent deformations and is universal at the infinitesimal level (see, e.g., [18]). An explicit construction of versal deformation has been given for Lie [3, 4], associative, infinity [5], and Leibniz algebras $[6]$.

The work was supported by INSA and by the Hungarian OTKA grant K77757.

Received January 18, 2013, revised October 21, 2013; published on May 15, 2014.

2010 Mathematics Subject Classification: 18D50, 17Axx, 18G50.

Key words and phrases: operad, algebra, cohomology, versal, deformation, obstruction, convolution Lie algebra, Maurer-Cartan element.

Article available at http://dx.doi.org/10.4310/HHA.2014.v16.n1.a11

Copyright (C) 2014, International Press. Permission to copy for private use granted. 
The aim of this paper is to show that the above method of construction of versal deformations of a particular type of algebra can be extended to construct versal deformations of an algebra over any quadratic operad. For this it is necessary to develop the relevant operadic tools. To this end we develop an obstruction theory for extending a given deformation, of an algebra over a quadratic operad with a finitedimensional local commutative algebra base $\mathcal{R}$, to a deformation over a suitable extension of $\mathcal{R}$ using the operadic calculus as developed in [13, Chapter 12].

The paper is organized as follows: In Section 2 we briefly review the basic definitions and results involving algebras over a quadratic operad, and the associated deformation complex necessary for our purpose, and we fix the notation that we follow throughout the text. In Section 3 we develop the deformation theory of algebras over an operad with local commutative algebra base. In Section 4 we study infinitesimal deformations and their properties. Given a deformation $\lambda$ of a $\mathcal{P}$-algebra $A$, with a finite-dimensional base $\mathcal{R}$ and a local abelian extension $0 \rightarrow M \rightarrow \mathcal{R}^{\prime} \rightarrow \mathcal{R} \rightarrow 0$ of $\mathcal{R}$ by an $\mathcal{R}$-module $M$ with $\operatorname{dim}_{\mathcal{R}} M<\infty$, we address in Section 5 the question of extending $\lambda$ to a deformation of $A$ with base $\mathcal{R}^{\prime}$. It turns out that, in general, this extension is not always possible. The associated obstruction can be interpreted as a 2 -cochain in the deformation complex associated to $A$. We prove that obstructions are 2-cocycles and that vanishing of the associated cohomology class is a necessary and sufficient condition for extending a given deformation to a deformation over a larger base. The results of Section 5 are crucial in order to construct a versal deformation of an algebra over a quadratic operad, which is the content of the next section. In Section 6 we introduce the notion of formal deformation of algebras over a quadratic operad with a complete local algebra base, and we define the notion of versal deformation. Finally, we give a construction of a versal deformation of an algebra over a quadratic operad, using the results developed in Sections 4 and 5.

\section{Preliminaries on operads and operadic cohomology}

In this section we recall some basic definitions and results about (algebraic) operads and the deformation complex of an algebra over a quadratic operad as in [13]. General references for operads and related results are $[\mathbf{9}, \mathbf{1 0}, \mathbf{1 3}, \mathbf{1 4}, \mathbf{1 5}, \mathbf{1 6}, \mathbf{1 7}]$.

The symbol $\mathbb{N}$ denotes the set of non-negative integers. Throughout this paper, we work over a fixed field $\mathbf{k}$ of characteristic 0 . We denote the category of vector spaces over $\mathbf{k}$ by Vect. The tensor product of vector spaces over $\mathbf{k}$ is denoted by $\otimes$. For any positive integer $n, \mathbb{S}_{n}$ denotes the group of permutations on $n$ elements. For any map $f: \otimes_{i=1}^{n} E_{i} \rightarrow F, f\left(x_{1}, \cdots, x_{n}\right)$ will stand for $f\left(x_{1} \otimes \cdots \otimes x_{n}\right)$.

\subsection{S-module}

Definition 2.1. An $\mathbb{S}$-module over $\mathbf{k}$ is a family

$$
M=\{M(0), M(1), \cdots, M(n), \cdots\}
$$

of right $\mathbf{k}\left[\mathbb{S}_{n}\right]$ modules $M(n)$. An $\mathbb{S}$-module $M$ is finite dimensional if $M(n)$ is finite dimensional for all $n$. A morphism $f: M \longrightarrow N$ between two $\mathbb{S}$-modules $M$ and $N$ is a family of maps $f(n): M(n) \longrightarrow N(n)$ that are $\mathbb{S}_{n}$-equivariant for all $n$.

Note that to every $\mathbb{S}$-module $M$ is associated a functor, called the Schur functor, 
$\widetilde{M}:$ Vect $\longrightarrow$ Vect such that $\widetilde{M}(V)=\oplus_{n \geqslant 0} M(n) \otimes_{\mathbb{S}_{n}} V^{\otimes n}$, where the left action of $\mathbb{S}_{n}$ on $V^{\otimes n}$ is given by $\sigma\left(v_{1}, v_{2}, \cdots, v_{n}\right)=\left(v_{\sigma^{-1}(1)}, v_{\sigma^{-1}(2)}, \cdots, v_{\sigma^{-1}(n)}\right)$.

The tensor product of two $\mathbb{S}$-modules is the $\mathbb{S}$-module $M \otimes N$ defined by

$$
(M \otimes N)(n)=\oplus_{i+j=n} I n d_{\mathbb{S}_{i} \times \mathbb{S}_{j}}^{\mathbb{S}_{n}} M(i) \otimes N(j) .
$$

The composite of two $\mathbb{S}$-modules $M$ and $N$ is the $\mathbb{S}$-module

$$
M \circ N=\oplus_{n \geqslant 0} M(n) \otimes N^{\otimes n},
$$

where $N^{\otimes n}$ is the tensor product of $n$ copies of the $\mathbb{S}$-module $N$. The category ( $\mathbb{S}-\mathrm{Mod}, \circ, I)$ is a monoidal category with respect to this composite product, where $I$ is the $\mathbb{S}$-module $(0, \mathbf{k}, 0, \cdots)$.

Recall that the composite of the two $\mathbb{S}$-modules $M$ and $N$ satisfies the formula

$$
(\widetilde{M \circ N})=\widetilde{M} \circ \widetilde{N}
$$

where on the right-hand side the symbol o stands for the composition of functors.

\subsection{Operads and cohomology}

Definition 2.2. A symmetric operad $\mathcal{P}=(\mathcal{P}, \gamma, \eta)$ is a monoid in the monoidal category $(\mathbb{S}$-Mod, o, $I)$. Explicitly, a symmetric operad $\mathcal{P}=(\mathcal{P}, \gamma, \eta)$ is an $\mathbb{S}$-module $\mathcal{P}=\{\mathcal{P}(n)\}_{n \geqslant 0}$ endowed with morphisms of $\mathbb{S}$-modules

$$
\gamma: \mathcal{P} \circ \mathcal{P} \longrightarrow \mathcal{P}
$$

called the composition map, and

$$
\eta: I \longrightarrow \mathcal{P}
$$

called the unit map, which makes $\mathcal{P}$ into a monoid.

Throughout the paper, by an operad we shall mean a symmetric operad.

Definition 2.3. Let $\mathcal{P}, \mathcal{Q}$ be operads. A morphism of operads from $\mathcal{P}$ to $\mathcal{Q}$ is a morphism of $\mathbb{S}$-modules $\alpha: \mathcal{P} \longrightarrow \mathcal{Q}$ that is compatible with the composition maps.

Example 2.4. Let $V$ be a vector space over $\mathbf{k}$, and for every $n \in \mathbb{N}$ let $\operatorname{End}_{V}(n)=$ $\operatorname{Hom}_{\mathbf{k}}\left(V^{\otimes n}, V\right)$. Then $\operatorname{End}_{V}=\left\{\operatorname{End}_{V}(n), n \in \mathbb{N}\right\}$ is naturally a symmetric operad, called the endomorphism operad of $V$.

Definition 2.5. Let $\mathcal{P}$ be an operad. A $\mathcal{P}$-algebra, or an algebra over $\mathcal{P}$, denoted by $(A, \alpha)$, is a vector space $A$ over $\mathbf{k}$, equipped with a morphism of operads $\alpha: \mathcal{P} \rightarrow$ $\operatorname{End}_{A}$.

A morphism of $\mathcal{P}$-algebras $\phi:(A, \alpha) \longrightarrow(B, \beta)$ is a k-linear map $\phi: A \longrightarrow B$ such that for any $a_{1}, \cdots, a_{n} \in A$ and $\mu \in \mathcal{P}(n)$,

$$
\phi\left(\alpha(\mu)\left(a_{1}, \cdots, a_{n}\right)\right)=\beta(\mu)\left(\phi\left(a_{1}\right), \cdots, \phi\left(a_{n}\right)\right) .
$$

Definition 2.6. The free operad over the $\mathbb{S}$-module $M$ is an operad $\mathcal{F}(M)$ equipped with an $\mathbb{S}$-module morphism $\eta(M): M \longrightarrow \mathcal{F}(M)$ that satisfies the following universal condition: any $\mathbb{S}$-module morphism $f: M \longrightarrow \mathcal{P}$, where $\mathcal{P}$ is an operad, extends uniquely to an operad morphism $\tilde{f}: \mathcal{F}(M) \longrightarrow \mathcal{P}$, that is, $\tilde{f} \circ \eta(M)=f$. 
The functor $\mathcal{F}$ from the category of $\mathbb{S}$-modules to the category of $\mathbf{k}$-operads is left adjoint to the forgetful functor from the category of $\mathbf{k}$-operads to $\mathbb{S}$-modules. An explicit construction of the free operad can be found in $[\mathbf{2}]$.

Definition 2.7. Let $\mathcal{P}$ be an operad. An ideal of $\mathcal{P}$ is a sub- $\mathbb{S}$-module $\mathcal{I}$ of $\mathcal{P}$ such that the operad structure of $\mathcal{P}$ passes on to the quotient $\mathcal{P} / \mathcal{I}$.

Let $E$ be an $\mathbb{S}$-module, and $R$ be a sub-S- -module such that $R \subseteq \mathcal{F}(E)^{(2)}$, where $\mathcal{F}(E)^{(2)}$ is the graded sub-S-module of the free operad $\mathcal{F}(E)$, which is spanned by the composites of two elements of $E$ [13, Section 5.5.3]. Such a pair $(E, R)$ is called a quadratic data.

Definition 2.8. Given a quadratic data $(E, R)$, let

$$
\mathcal{P}(E, R)=\mathcal{F}(E) /(R)
$$

be the quotient of the free operad $\mathcal{F}(E)$ over $E$ by the operadic ideal $(R)$ generated by $R$. Then the operad $\mathcal{P}(E, R)$ is called the quadratic operad associated to the pair $(E, R)$.

Definition 2.9. An $\mathbb{S}$-module $\mathcal{C}$ is a cooperad if it is a comonoid in the monoidal category ( $\mathbb{S}$-mod,,$I)$. A cofree cooperad on an $\mathbb{S}$-module $M$ is the cooperad $\mathcal{F}^{c}(M)$, which is cofree in the category of conilpotent cooperads.

Recall that the Koszul dual cooperad of a quadratic operad is defined as follows:

Definition 2.10. The quadratic cooperad $\mathcal{C}(E, R)$ associated to the quadratic data $(E, R)$ is the sub-cooperad of the cofree cooperad $\mathcal{F}^{c}(E)$ that is universal among the sub-cooperads of $\mathcal{F}^{c}(E)$ such that the following composite is 0 ,

$$
\mathcal{C} \hookrightarrow \mathcal{F}^{c}(E) \rightarrow \mathcal{F}^{c}(E)^{(2)} /(R) .
$$

The Koszul dual cooperad of the quadratic operad $\mathcal{P}(E, R)$ is the quadratic cooperad

$$
\mathcal{P}^{\mathrm{i}}:=\mathcal{C}\left(s E, s^{2} R\right)
$$

where $s E$ denotes the $\mathbb{S}$-module $E$ whose degree is shifted by 1 .

We recall the definition of the deformation complex of an algebra over a quadratic operad [13].

Let $A$ be a $\mathcal{P}$-algebra, where $\mathcal{P}=\mathcal{P}(E, R)$ is a quadratic operad. Let

$$
\mathfrak{g}=\mathfrak{g}_{\mathcal{P}, A}=\left(\operatorname{Hom}_{\mathbb{S}}\left(\mathcal{P}^{\mathrm{i}}, \operatorname{End}_{A}\right),[,], \partial\right)
$$

be the convolution dg Lie algebra associated to $A$. Since $A$ is concentrated in degree 0 , the cohomological degree on the differential graded Lie algebra

$$
\mathfrak{g}: \operatorname{Hom}(A, A) \stackrel{\partial}{\longrightarrow} \operatorname{Hom}_{\mathbb{S}}\left(\mathcal{P}^{\mathbf{i}^{(1)}}, \operatorname{End}_{A}\right) \stackrel{\partial}{\longrightarrow} \operatorname{Hom}_{\mathbb{S}}\left(\mathcal{P}^{\mathbf{i}(2)}, \operatorname{End}_{A}\right) \cdots
$$

is induced by the weight grading. Thus $\operatorname{Hom}(A, A)$ is the 0th cochain module and $\operatorname{Hom}_{\mathbb{S}}\left(\mathcal{P i}^{(n)}, \operatorname{End}_{A}\right)$ is the $n$th cochain module. Moreover, since $\mathcal{P}$ is homogeneous quadratic, the coboundary map $\partial$ in the above complex is null. We note that the set of $\mathcal{P}$-algebra structures on a space $A$ is in one-to-one correspondence with the set of Maurer-Cartan elements of degree 1 of $\mathfrak{g}$, i.e., all elements $\phi$ in $\operatorname{Hom}_{\mathbb{S}}\left(\mathcal{P}^{\mathrm{i}}{ }^{(1)}, \operatorname{End}_{A}\right)$ 
satisfying $[\phi, \phi]=0$. Given such an element $\phi$, one can define a differential $\partial_{\phi}$ on the Lie algebra $\mathfrak{g}$, which makes it into a differential graded Lie algebra. The differential $\partial_{\phi}$ is called the twisted differential, and $\left.\mathfrak{g}^{\phi}=\mathfrak{g}_{\mathcal{P}, A}^{\phi}=\left(\operatorname{Hom}_{\mathbb{S}}\left(\mathcal{P} \mathrm{i}, \operatorname{End}_{A}\right)\right),[],, \partial_{\phi}\right)$ is called the twisted differential graded Lie algebra. The twisted differential $\partial_{\phi}$ is given by: $\partial_{\phi}(f)=[\phi, f]$. The underlying cochain complex of this twisted dg Lie algebra is the deformation complex that we intend to work with. We end this section with the following definition.

Definition 2.11. For any $\mathcal{P}$-algebra $(A, \pi)$ where $\mathcal{P}$ is a quadratic operad, we define

$$
H_{\mathcal{P}}^{*}(A):=H_{*}\left(\mathfrak{g}^{\pi}\right)=H_{*}\left(\mathfrak{g}, \partial_{\pi}\right) .
$$

\section{Deformations}

Let $\mathcal{R}$ be a commutative local unital algebra with unit $1_{\mathcal{R}}$ over $\mathbf{k}$. Let $\epsilon: \mathcal{R} \rightarrow$ $\mathbf{k}, \epsilon\left(1_{\mathcal{R}}\right)=1$ be the canonical augmentation map, and let $\mathfrak{M}=k e r(\epsilon)$ be the unique maximal ideal in $\mathcal{R}$. In this section, we study the notion of deformation of an algebra over a quadratic operad with base $\mathcal{R}$, and its properties.

Let $\mathcal{P}=\mathcal{P}(E, R)$ be a quadratic operad. We will denote by $\mathcal{P}_{\mathcal{R}}$ the operad that is obtained by extension of $\mathcal{P}$ to the category of modules over $\mathcal{R}$; in other words, $\mathcal{P}_{\mathcal{R}}(n)=\mathcal{R} \otimes \mathcal{P}(n)$ for all $n \in \mathbb{N}$. Let $(A, \pi)$ be a $\mathcal{P}$-algebra. Let $A_{\mathcal{R}}=\mathcal{R} \otimes A$ denote the extension of $A$. Then $A_{\mathcal{R}}$ can be viewed as a $\mathcal{P}_{\mathcal{R}}$-algebra by extending $\pi: \mathcal{P} \longrightarrow$ $\operatorname{End}_{A}$ to $\pi_{\mathcal{R}}: \mathcal{P}_{\mathcal{R}} \longrightarrow \operatorname{End}_{A_{\mathcal{R}}}$, since

$$
\operatorname{Hom}_{\mathcal{R}}\left(A_{\mathcal{R}}^{\otimes_{\mathcal{R}} n}, A_{\mathcal{R}}\right) \cong \mathcal{R} \otimes \operatorname{Hom}_{\mathbf{k}}\left(A^{\otimes n}, A\right) .
$$

Moreover, $A=\mathbf{k} \otimes A$ can be viewed as a $\mathcal{P}_{\mathcal{R}}$-algebra by considering $A$ as a module over $\mathcal{R}$ via $\epsilon$; that is, the $\mathcal{R}$-module structure on $A$ is given by $r \cdot a=\epsilon(r) a$, for $r \in \mathcal{R}$ and $a \in A$.

Definition 3.1. A deformation $\lambda$ of a $\mathcal{P}$-algebra $(A, \pi)$ with base $(\mathcal{R}, \mathfrak{M})$ is a morphism of operads $\lambda: \mathcal{P}_{\mathcal{R}} \longrightarrow \operatorname{End}_{A_{\mathcal{R}}}$ such that $(\epsilon \otimes \mathrm{Id}): A_{\mathcal{R}} \longrightarrow \mathbf{k} \otimes A \cong A$ is a $\mathcal{P}_{\mathcal{R}^{-}}$ algebra morphism.

In other words, a deformation of $(A, \pi)$ with base $(\mathcal{R}, \mathfrak{M})$ is a $\mathcal{P}_{\mathcal{R}}$-algebra structure on $A_{\mathcal{R}}$ that reduces to $\pi$, modulo $\mathfrak{M}: A \cong \mathbf{k} \otimes_{\mathcal{R}}(\mathcal{R} \otimes A)$.

We have the following result [13, Proposition 12.2.6].

Proposition 3.2. For any $\mathcal{P}$-algebra $(A, \pi)$, the set of all deformations of $(A, \pi)$ with base $(\mathcal{R}, \mathfrak{M})$ is in one-to-one correspondence with the set of all Maurer-Cartan elements in the dg Lie algebra $\mathfrak{M} \otimes \mathfrak{g}^{\pi}$.

Definition 3.3. Suppose $\lambda_{1}$ and $\lambda_{2}$ are two deformations of a $\mathcal{P}$-algebra $(A, \pi)$ with the same base $(\mathcal{R}, \mathfrak{M})$. They are said to be equivalent if there exists a $\mathcal{P}_{\mathcal{R}}$-algebra isomorphism $\phi:\left(A_{\mathcal{R}}, \lambda_{1}\right) \longrightarrow\left(A_{\mathcal{R}}, \lambda_{2}\right)$ such that

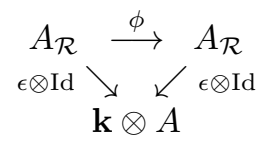

commutes. 
Definition 3.4. Let $\mathcal{R}$ be a complete local algebra, $\mathcal{R}=\lim \left(\mathcal{R} / \mathfrak{M}^{n}\right), \mathfrak{M}$ denoting the maximal ideal in $\mathcal{R}$. A formal deformation of a $\mathcal{P}$-algebra $(A, \pi)$ with base $(\mathcal{R}, \mathfrak{M})$ is a $\mathcal{P}_{\mathcal{R}}$-algebra structure on the completed tensor product $\mathcal{R} \widehat{\otimes} A=\overleftarrow{\lim }\left(\left(\mathcal{R} / \mathfrak{M}^{n}\right) \otimes A\right)$ such that $\epsilon \widehat{\otimes} \mathrm{Id}: \mathcal{R} \widehat{\otimes} A \longrightarrow \mathbf{k} \otimes A \cong A$ is a $\mathcal{P}_{\mathcal{R}}$-algebra morphism.

Example 3.5. Let $\mathcal{R}=\mathbf{k}[[t]]$ be the ring of formal power series with coefficients in $\mathbf{k}$. Then a formal deformation of a $\mathcal{P}$-algebra $(A, \pi)$ over $\mathcal{R}$ is precisely the formal "1-parameter" deformation of $(A, \pi)$.

Definition 3.6. Let $\lambda$ be a deformation of the $\mathcal{P}$-algebra $(A, \pi)$ with base $(\mathcal{R}, \mathfrak{M})$ and augmentation $\epsilon: \mathcal{R} \longrightarrow \mathbf{k}$. Let $\mathcal{R}^{\prime}$ be another commutative local algebra with unit, and augmentation $\epsilon^{\prime}: \mathcal{R}^{\prime} \longrightarrow \mathbf{k}$ with $\operatorname{Ker}\left(\epsilon^{\prime}\right)=\mathfrak{M}^{\prime}$. Let $f: \mathcal{R} \longrightarrow \mathcal{R}^{\prime}$ be an algebra homomorphism with $f\left(1_{\mathcal{R}}\right)=1_{\mathcal{R}^{\prime}}$. Then $\epsilon^{\prime} \circ f=\epsilon$.

Consider $\mathcal{R}^{\prime}$ as an $\mathcal{R}$-module via the map $f: r^{\prime} \cdot r=r^{\prime} f(r)$ so that

$$
\mathcal{R}^{\prime} \otimes A=\left(\mathcal{R}^{\prime} \otimes_{\mathcal{R}} \mathcal{R}\right) \otimes A=\mathcal{R}^{\prime} \otimes_{\mathcal{R}}(\mathcal{R} \otimes A) .
$$

Then the push-out of the deformation $\lambda$ is the deformation $f_{*} \lambda$ of $(A, \pi)$ with base $\left(\mathcal{R}^{\prime}, \mathfrak{M}^{\prime}\right)$, defined by

$$
\begin{aligned}
f_{*} \lambda\left(1_{\mathcal{R}^{\prime}} \otimes \mu\right)\left\{r_{1}^{\prime} \otimes_{\mathcal{R}}\left(r_{1} \otimes a_{1}\right), r_{2}^{\prime} \otimes_{\mathcal{R}}\left(r_{2} \otimes a_{2}\right), \cdots, r_{n}^{\prime} \otimes_{\mathcal{R}}\left(r_{n} \otimes a_{n}\right)\right\} \\
=r_{1}^{\prime} r_{2}^{\prime} \cdots r_{n}^{\prime} \otimes_{\mathcal{R}} \lambda\left(1_{\mathcal{R}} \otimes \mu\right)\left(r_{1} \otimes a_{1}, r_{2} \otimes a_{2}, \cdots, r_{n} \otimes a_{n}\right), \quad \mu \in \mathcal{P}^{\mathrm{i}(1)}(n) .
\end{aligned}
$$

It is straightforward to see that $f_{*} \lambda$ is a deformation of $(A, \pi)$ with base $\left(\mathcal{R}^{\prime}, \mathfrak{M}^{\prime}\right)$.

Remark 3.7. Note that by the proof of Proposition 3.2, as in [13], if $\lambda$ is a deformation of the $\mathcal{P}$-algebra $(A, \pi)$ with a base $(\mathcal{R}, \mathfrak{M})$, then $\lambda$ can be expressed as

$$
\lambda=\pi+\sum_{i=1}^{s} m_{i} \otimes \phi_{i}
$$

where $\pi$ is a Maurer-Cartan element of $\mathfrak{g}^{\pi}$ and $\sum_{i=1}^{s} m_{i} \otimes \phi_{i}$ is a Maurer-Cartan element of $\mathfrak{M} \otimes \mathfrak{g}^{\pi}$. Also, the push-out $f_{*} \lambda$ is given by

$$
f_{*} \lambda=\pi+\sum_{i=1}^{s} f\left(m_{i}\right) \otimes \phi_{i},
$$

where $\sum_{i=1}^{s} f\left(m_{i}\right) \otimes \phi_{i}$ is a Maurer-Cartan element of $\mathfrak{M}^{\prime} \otimes \mathfrak{g}^{\pi}$.

Definition 3.8. A deformation $\lambda$ of $(A, \pi)$ with base $(\mathcal{R}, \mathfrak{M})$ is called infinitesimal if, in addition, $\mathfrak{M}^{2}=0$.

To consider the equivalence of infinitesimal deformations, cohomology comes into play naturally.

Let $\lambda$ be an infinitesimal deformation of $(A, \pi)$, with base $(\mathcal{R}, \mathfrak{M})$ such that

$$
\lambda=\pi+\sum_{i=1}^{s} m_{i} \otimes \phi_{i}
$$

Let $\xi \in \mathfrak{M}^{\prime}=\operatorname{Hom}_{\mathbf{k}}(\mathfrak{M}, \mathbf{k})$. Clearly, $\xi$ can be viewed as an element of $\operatorname{Hom}_{\mathbf{k}}(\mathcal{R}, \mathbf{k})$ with $\xi\left(1_{\mathcal{R}}\right)=0$.

Define a 1-cochain $\alpha_{\lambda, \xi}$ of $\mathfrak{g}^{\pi}$ by

$$
\alpha_{\lambda, \xi}=(\xi \otimes \mathrm{Id}) \lambda .
$$


Theorem 3.9. For any infinitesimal deformation $\lambda$ of a $\mathcal{P}$-algebra $(A, \pi), \alpha_{\lambda, \xi}$ is a 1-cocycle.

Proof. As in Remark 3.7, we write $\lambda=\pi+\sum_{i=1}^{s} m_{i} \otimes \phi_{i}$. Then, using the fact that $\xi\left(1_{\mathcal{R}}\right)=0$, the 1-cochain $\alpha_{\lambda, \xi}$ can be expressed as

$$
\alpha_{\lambda, \xi}=(\xi \otimes \mathrm{Id}) \lambda=(\xi \otimes \mathrm{Id})\left(\pi+\sum_{i=1}^{s} m_{i} \otimes \phi_{i}\right)=\sum_{i=1}^{s} \xi\left(m_{i}\right) \phi_{i} .
$$

The fact that $\sum_{i=1}^{s} m_{i} \otimes \phi_{i}$ ia a Maurer-Cartan element in $\mathfrak{M} \otimes \mathfrak{g}^{\pi}$, and the fact that $\mathfrak{M}^{2}=0$, implies that

$$
\sum_{i=1}^{s} m_{i} \otimes\left[\phi_{i}, \pi\right]=0
$$

Therefore,

$$
\sum_{i=1}^{s} \xi\left(m_{i}\right)\left[\pi, \phi_{i}\right]=(\xi \otimes \mathrm{Id})\left(\sum_{i=1}^{s} m_{i} \otimes\left[\pi, \phi_{i}\right]\right)=0 .
$$

Using the above observations, we deduce that

$$
\partial_{\pi}\left(\alpha_{\lambda, \xi}\right)=\left[\pi, \alpha_{\lambda, \xi}\right]=\left[\pi, \sum_{i=1}^{s} \xi\left(m_{i}\right) \phi_{i}\right]=\sum_{i=1}^{s} \xi\left(m_{i}\right)\left[\pi, \phi_{i}\right]=0 .
$$

Let us define for $\xi \in \mathfrak{M}^{\prime}$ the cohomology class of the cocycle $\alpha_{\lambda, \xi}$ by $a_{\lambda, \xi}$. The correspondence $\xi \longmapsto a_{\lambda, \xi}$ defines a map

$$
a_{\lambda}: \mathfrak{M}^{\prime} \longrightarrow H_{\mathcal{P}}^{1}(A) .
$$

Theorem 3.10. Let $\lambda_{1}$ and $\lambda_{2}$ be two infinitesimal deformations of $(A, \pi)$ with base $(\mathcal{R}, \mathfrak{M})$. Assume that $\mathcal{R}$ is of finite dimension. Then the deformations $\lambda_{1}$ and $\lambda_{2}$ are equivalent iff $\alpha_{\lambda_{1}, \xi}$ and $\alpha_{\lambda_{2}, \xi}$ represent the same cohomology class; that is, $a_{\lambda_{1}, \xi}=$ $a_{\lambda_{2}, \xi}$ for $\xi \in \mathfrak{M}^{\prime}$.

Proof. This result is proved in [13, Theorem 12.2.7] for $R=\mathbf{k}[t] /\left(t^{2}\right)$. The present theorem is proved by following the same idea. We only give the essential steps.

Let $\left\{m_{i}\right\}_{1 \leqslant i \leqslant r}$ be a basis of $\mathfrak{M}$, and let $\left\{\xi_{i}\right\}_{1 \leqslant i \leqslant r}$ be the dual basis of $\mathfrak{M}^{\prime}$. By definition, $\lambda_{1}$ and $\lambda_{2}$ are equivalent if, and only if, there exists a $\mathcal{P}_{\mathcal{R}}$-algebra isomorphism

$$
\rho: A_{\mathcal{R}} \longrightarrow A_{\mathcal{R}} \text {, such that }(\epsilon \otimes \mathrm{Id}) \circ \rho=\epsilon \otimes \mathrm{Id} .
$$

Since $A_{\mathcal{R}}=\mathcal{R} \otimes A=(\mathbf{k} \oplus \mathfrak{M}) \otimes A \cong A \oplus(\mathfrak{M} \otimes A)$, the isomorphism $\rho$ can be written as $\rho=\rho_{1}+\rho_{2}$, where $\rho_{1}: A \longrightarrow A$ and $\rho_{2}: A \longrightarrow \mathfrak{M} \otimes A$.

By compatibility (3.3), we get $\rho_{1}=\mathrm{Id}$. Using the adjunction property of tensor products, we have

$$
\operatorname{Hom}_{\mathbf{k}}(A ; \mathfrak{M} \otimes A) \cong \mathfrak{M} \otimes \operatorname{Hom}_{\mathbf{k}}(A, A) \cong \operatorname{Hom}_{\mathbf{k}}\left(\mathfrak{M}^{\prime} ; \operatorname{Hom}_{\mathbf{k}}(A, A)\right),
$$

where the isomorphisms are given by

$$
\rho_{2} \longmapsto \sum_{1}^{r} m_{i} \otimes \phi_{i} \longmapsto \sum_{i}^{r} \chi_{i}
$$

Here $\phi_{i}=\left(\xi_{i} \otimes \mathrm{id}\right) \circ \rho_{2}, \chi_{i}\left(\xi_{j}\right)=\delta_{i, j} \phi_{i}$. 
Thus we can write

$$
\rho=\mathrm{Id}+\sum_{1}^{r} m_{i} \otimes \phi_{i} .
$$

Recall that $\rho$ is a $\mathcal{P}_{\mathcal{R}}$-algebra morphism iff

$$
\rho\left(\lambda_{1}\left(1_{\mathcal{R}} \otimes \mu\right)\right)=\lambda_{2}\left(1_{\mathcal{R}} \otimes \mu\right)(\rho, \rho, \cdots, \rho),
$$

$\mu \in \mathcal{P}^{(1)}(n)$, where on the right-hand side we have $n$ many copies of $\rho$.

Let us set $\psi_{i}^{k}=\alpha_{\lambda_{k}, \xi_{i}}, i=1,2, \ldots, r$, and $k=1,2$. Then we have

$$
\lambda_{k}\left(1_{\mathcal{R}} \otimes \mu\right)=\pi(\mu)+\sum_{1}^{r} m_{i} \otimes \psi_{i}^{k}(\mu)
$$

for all $\mu \in \mathcal{P}^{(1)}$. We explicitly write both sides of the equation (3.5), using the fact that the deformations involved are infinitesimal:

$$
\begin{array}{r}
\lambda_{2}\left(1_{\mathcal{R}} \otimes \mu\right)(\rho, \rho, \cdots, \rho) \\
=\pi(\mu)+\sum_{i=1}^{r} m_{i} \otimes \psi_{i}^{2}(\mu)+\sum_{k=1}^{n} \sum_{i=1}^{r} m_{i} \otimes\left(\pi(\mu)\left(\operatorname{Id}, \operatorname{Id}, \cdots, \phi_{i}, \operatorname{Id}, \cdots\right)\right),
\end{array}
$$

where in the last summand $\phi_{i}$ is in the $k$ th slot.

On the other hand,

$$
\begin{aligned}
\rho\left(\lambda_{1}\left(1_{\mathcal{R}} \otimes \mu\right)\right) & =\rho\left(\pi(\mu)+\sum_{1}^{r} m_{i} \otimes \psi_{i}^{1}(\mu)\right) \\
& =\pi(\mu)+\sum_{1}^{r} m_{i} \otimes \phi_{i} \circ \pi(\mu)+\sum_{1}^{r} m_{i} \otimes \psi_{i}^{1}(\mu) .
\end{aligned}
$$

It follows from (3.5) that

$$
\sum_{1}^{r} m_{i} \otimes\left(\psi_{i}^{2}-\psi_{i}^{1}\right)+\sum_{1}^{r} m_{i} \otimes \partial_{\pi} \phi_{i}=0 .
$$

Hence,

$$
\partial_{\pi} \phi_{i}=\psi_{i}^{2}-\psi_{i}^{1}=\alpha_{\lambda_{2}, \xi_{i}}-\alpha_{\lambda_{1}, \xi_{i}} \quad \text { for all } i=1, \ldots, r .
$$

So, $a_{\lambda_{1}, \xi}=a_{\lambda_{2}, \xi}$, for all $\xi \in \mathfrak{M}^{\prime}$.

Let $\mathcal{R}$ be a local algebra with maximal ideal $\mathfrak{M}$. Then $\mathcal{R} / \mathfrak{M}^{2}$ is local with maximal ideal $\mathfrak{M} / \mathfrak{M}^{2}$ and $\left(\mathfrak{M} / \mathfrak{M}^{2}\right)^{2}=0$. Let $q: \mathcal{R} \longrightarrow \mathcal{R} / \mathfrak{M}^{2}$ be the projection map. Let $\lambda$ be a deformation of $(A, \pi)$ with base $(\mathcal{R}, \mathfrak{M})$. Then the deformation $q_{*} \lambda$ is infinitesimal and we have a map

$$
a_{q_{*} \lambda}:\left(\mathfrak{M} / \mathfrak{M}^{2}\right)^{\prime} \longrightarrow H_{\mathcal{P}}^{1}(A) .
$$

Definition 3.11. The dual space $\left(\mathfrak{M} / \mathfrak{M}^{2}\right)^{\prime}$ is called the tangent space of $\mathcal{R}$ and is denoted by $T \mathcal{R}$. The mapping

$$
a_{q_{*} \lambda}:\left(\mathfrak{M} / \mathfrak{M}^{2}\right)^{\prime} \longrightarrow H_{\mathcal{P}}^{1}(A)
$$

is called the differential of $\lambda$ and is denoted by $d \lambda$. In particular, if $\mathfrak{M}^{2}=0$, then the differential $d \lambda$ of the infinitesimal deformation $\lambda$ is the map $a_{\lambda}$. 
Corollary 3.12. If two deformations $\lambda_{1}$ and $\lambda_{2}$ of a $\mathcal{P}_{\mathcal{R}}$-algebra $(A, \pi)$ are equivalent, then their differentials are equal.

\section{Universal Infinitesimal deformation}

In this section we construct a specific example of an infinitesimal deformation of a $\mathcal{P}$-algebra satisfying finite dimensionality of the first cohomology module. We shall also prove a fundamental property of this deformation. In the last section we will see that this infinitesimal deformation is the first step of an inductive construction of a versal deformation.

Let $(A, \pi)$ be a given $\mathcal{P}$-algebra satisfying the condition $\operatorname{dim} H_{\mathcal{P}}^{1}(A)<\infty$. Let us denote $H_{\mathcal{P}}^{1}(A)$ by $\mathbb{H}$. Consider the $\mathbf{k}$-algebra $C_{1}=\mathbf{k} \oplus \mathbb{H}^{\prime}$ with the following structure:

$$
\left(k_{1}, h_{1}\right) \cdot\left(k_{2}, h_{2}\right)=\left(k_{1} k_{2}, k_{1} h_{2}+k_{2} h_{1}\right) .
$$

Clearly, $C_{1}$ is local with maximal ideal $\mathfrak{M}=\mathbb{H}^{\prime}$ and $\mathfrak{M}^{2}=0$.

Fix a homomorphism

$$
\sigma: \mathbb{H} \longrightarrow \mathfrak{g}_{\mathcal{P}, A}^{1}=\operatorname{Hom}_{\mathbb{S}}\left(\mathcal{P}^{\mathrm{i}}(1), \operatorname{End}_{A}\right)
$$

that takes a cohomology class into a representative cocycle. We note that

$$
\mathbb{H}^{\prime} \otimes A=\operatorname{Hom}_{\mathbf{k}}\left(H_{\mathcal{P}}^{1}(A), \mathbf{k}\right) \otimes A \cong \operatorname{Hom}_{\mathbf{k}}\left(H_{\mathcal{P}}^{1}(A), A\right)
$$

and

$$
A_{\mathcal{C}_{1}}=C_{1} \otimes A \cong\left(\mathbf{k} \oplus \mathbb{H}^{\prime}\right) \otimes A \cong A \oplus \operatorname{Hom}_{\mathbf{k}}(\mathbb{H}, A) .
$$

Define a $\mathcal{P}_{C_{1}}$-algebra structure $\eta_{1}$ on $A_{C_{1}}$ by

$$
\eta_{1}=\pi+\sum_{i=1}^{n} h_{i}^{*} \otimes \sigma\left(h_{i}\right)
$$

as a map $C_{1} \otimes \mathcal{P}^{(1)} \longrightarrow\left(\mathbf{k} \oplus \mathbb{H}^{\prime}\right) \otimes \operatorname{End}_{A}=C_{1} \otimes \operatorname{End}_{A}$, where $\left\{h_{i}\right\}_{1 \leqslant i \leqslant n}$ denotes a finite basis of $\mathbb{H}$ and $\left\{h_{i}{ }^{*}\right\}_{1 \leqslant i \leqslant n}$ denotes the dual basis.

Proposition 4.1. For any homomorphism $\sigma: \mathbb{H} \longrightarrow \mathfrak{g}_{\mathcal{P}, A}^{1}=\operatorname{Hom}_{\mathbb{S}}\left(\mathcal{P}^{i(1)}, \operatorname{End}_{A}\right)$, $\left(A_{C_{1}}, \eta_{1}\right)$ is a $\mathcal{P}_{C_{1}}$-algebra.

Proof. We need to show that $\eta_{1}$ is a Maurer-Cartan element in $C_{1} \otimes \mathfrak{g}^{\pi}$. The coboundary of the complex $C_{1} \otimes \mathfrak{g}^{\pi}$ is given by

$$
\bar{\partial}_{\pi}:=\operatorname{Id}_{C_{1}} \otimes \partial_{\pi}
$$

From (4.1),

$$
\eta_{1}=\pi+\sum_{i} h_{i}^{*} \otimes \sigma\left(h_{i}\right)
$$

So

$$
\left.\bar{\partial}_{\pi}\left(\eta_{1}\right)=\operatorname{Id}_{C_{1}} \otimes\left[\pi, \eta_{1}\right]=\operatorname{Id}_{C_{1}} \otimes[\pi, \pi]+\sum_{i} h_{i}^{*} \otimes\left[\pi, \sigma\left(h_{i}\right)\right]\right)=0,
$$

using the fact that $\sigma\left(h_{i}\right)$ is a 1-cocycle in $\mathfrak{g}^{\pi}$. 
Proposition 4.2. Up to isomorphism, the $\mathcal{P}_{C_{1}}$-algebra structures of $A_{C_{1}}$ does not depend on the choice of $\sigma$.

Proof. Let $\sigma^{\prime}: \mathbb{H} \longrightarrow \mathfrak{g}_{\mathcal{P}, A}^{1}$ be another choice of $\sigma$, and denote the corresponding $\mathcal{P}_{C_{1}}{ }^{-}$ algebra structure on $C_{1} \otimes A$ by $\eta^{\prime}$. Then for $h \in \mathbb{H}, \sigma(h)$ and $\sigma^{\prime}(h)$ are two 1-cocycles of $A$, representing the same cohomology class; that is, $\sigma(h)-\sigma^{\prime}(h)$ is a 1-coboundary. Let $\sigma(h)-\sigma^{\prime}(h)=\partial_{\pi}(\gamma(h))$, where $\gamma: \mathbb{H} \longrightarrow \operatorname{Hom}_{\mathbf{k}}(A, A)$. Here $\operatorname{Hom}_{\mathbf{k}}(A, A)$ is the 0 cochains of $\mathfrak{g}^{\pi}$. Using the identification

$$
C_{1} \otimes A \cong A \oplus \operatorname{Hom}_{\mathbf{k}}(\mathbb{H}, A),
$$

define a $C_{1}$-linear automorphism $\rho: C_{1} \otimes A \longrightarrow C_{1} \otimes A$ by $\rho(a, \phi)=(a, \bar{\phi})$, where $\bar{\phi}(h)=\phi(h)+\gamma(h)(a)$. We need to show that $\rho$ preserves the $\mathcal{P}_{C_{1}}$-algebra structure, that is,

$$
\rho\left(\eta_{1}\left(1_{C_{1}} \otimes \mu\right)\right)=\eta^{\prime}\left(1_{C_{1}} \otimes \mu\right) \circ(\rho, \rho, \cdots, \rho),
$$

where the number of copies of $\rho$ on the right-hand side is the same as the (homogeneous) degree of $\mu$ in $\mathcal{P}^{i^{(1)}}$. Now observe that, using the isomorphism

$$
\operatorname{Hom}_{\mathbf{k}}(\mathbb{H}, A) \cong \operatorname{Hom}_{\mathbf{k}}(\mathbb{H}, \mathbf{k}) \otimes A,
$$

we can rewrite the expression (4.1) of $\eta_{1}$ as

$$
\eta_{1}\left(1_{C_{1}} \otimes \mu\right)\left(\left(a_{1}, \phi_{1}\right), \cdots,\left(a_{n}, \phi_{n}\right)\right)=\left(\pi(\mu)\left(a_{1}, \cdots, a_{n}\right), \psi_{\mu}\right),
$$

where $\psi_{\mu}(h)=\sigma(h)(\mu)\left(a_{1}, \cdots, a_{n}\right)+\sum_{i} \pi(\mu)\left(a_{1}, \cdots, \phi_{i}(h), \cdots, a_{n}\right)$, where in the summation, $\phi_{i}$ is at the $i$ th slot. Similarly,

$$
\eta^{\prime}\left(1_{C_{1}} \otimes \mu\right)\left(\left(a_{1}, \phi_{1}\right), \cdots,\left(a_{n}, \phi_{n}\right)\right)=\left(\pi(\mu)\left(a_{1}, \cdots, a_{n}\right), \psi_{\mu}^{\prime}\right),
$$

where

$$
\begin{aligned}
\psi_{\mu}^{\prime}(h) & =\sigma^{\prime}(h)(\mu)\left(a_{1}, \cdots, a_{n}\right)+\sum_{i} \pi(\mu)\left(a_{1}, \cdots, \phi_{i}(h), \cdots, a_{n}\right) \\
& =\left(\sigma(h)-\partial_{\pi}(\gamma(h))\right)(\mu)\left(a_{1}, \cdots, a_{n}\right)+\sum_{i} \pi(\mu)\left(a_{1}, \cdots, \phi_{i}(h), \cdots, a_{n}\right) .
\end{aligned}
$$

We evaluate both sides of (4.2) using the expressions of $\eta_{1}, \eta^{\prime}$ as given above and the definitions of $\rho$ and the coboundary $\partial_{\pi}$, to get $\psi_{\mu}^{\prime}(h)-\psi_{\mu}(h)=0$, for all $h \in \mathbb{H}$. Then equation (4.2) holds.

The main property of the infinitesimal deformation $\eta_{1}$ is its universality in the class of infinitesimal deformations with finite-dimensional base.

Theorem 4.3. For any infinitesimal deformation $\lambda$ of the $\mathcal{P}$-algebra $A$ with a finitedimensional local base $(\mathcal{R}, \mathfrak{M})$, there exists a unique homomorphism $\phi: C_{1} \rightarrow \mathcal{R}$ such that $\lambda$ is equivalent to the push-out $\phi_{*} \eta_{1}$.

Proof. Let $a_{\lambda}: \mathfrak{M}^{\prime} \rightarrow H_{\mathcal{P}}^{1}(A)=\mathbb{H}$ denote the differential of $\lambda$,

$$
a_{\lambda}: \xi \mapsto a_{\lambda, \xi}=\left[\alpha_{\lambda, \xi}\right], \quad \xi \in \mathfrak{M}^{\prime} .
$$

Consider the map $\phi=\operatorname{Id} \oplus a_{\lambda}^{\prime}: \mathbf{k} \oplus \mathbb{H}^{\prime} \rightarrow \mathbf{k} \oplus \mathfrak{M}=\mathcal{R}$. Let $\left\{m_{i}\right\}_{i=1}^{r}$ be a basis of $\mathfrak{M}$, and let $\left\{\xi_{i}\right\}$ be the dual basis.

It is enough to show $\alpha_{\phi_{*} \eta_{1}}=\sigma \circ a_{\lambda}$ (Theorem 3.10). Let $\left\{h_{1}, \cdots, h_{n}\right\}$ be a basis of $\mathbb{H}$, and let $\left\{h_{1}{ }^{*}, \cdots, h_{n}{ }^{*}\right\}$ be the corresponding dual basis of $\mathbb{H}^{\prime}$. 
Then by Remark 3.7 and equation (3.2) we have

$$
\phi_{*} \eta_{1}=\pi+\sum_{j=1}^{n} \phi\left(h_{j}^{*}\right) \otimes \sigma\left(h_{j}\right) .
$$

Now,

$$
a_{\lambda}^{\prime}\left(h_{j}{ }^{*}\right)=\sum_{i=1}^{r} \xi_{i}\left(a_{\lambda}^{\prime}\left(h_{j}{ }^{*}\right)\right) m_{i} \quad \text { and } \quad a_{\lambda}\left(\xi_{i}\right)=\sum_{j=1}^{n} h_{j}{ }^{*}\left(a_{\lambda}\left(\xi_{i}\right)\right) h_{j} .
$$

Thus

$$
\begin{aligned}
\alpha_{\phi_{*} \eta_{1}}\left(\xi_{i}\right)\left(\mu ; a_{1}, \cdots, a_{n}\right) & \\
= & \left(\xi_{i} \otimes \mathrm{Id}\right) \phi_{*} \eta_{1}(\mu)\left(1_{\mathcal{R}} \otimes a_{1}, \cdots, 1_{\mathcal{R}} \otimes a_{n}\right) \\
& =\left(\xi_{i} \otimes \mathrm{Id}\right)\left(\pi(\mu)\left(a_{1}, \cdots, a_{n}\right)+\sum_{j=1}^{n} \phi\left(h_{j}{ }^{*}\right) \otimes \sigma\left(h_{j}\right)\left(\mu ; a_{1}, \cdots, a_{n}\right)\right) \\
& =\left(\xi_{i} \otimes \mathrm{Id}\right)\left(\sum_{j=1}^{n} a_{\lambda}^{\prime}\left(h_{j}{ }^{*}\right) \otimes \sigma\left(h_{j}\right)\left(\mu ; a_{1}, \cdots, a_{n}\right)\right) \\
& =\sum_{j=1}^{n} \xi_{i}\left(a_{\lambda}^{\prime}\left(h_{j}{ }^{*}\right)\right) \otimes \sigma\left(h_{j}\right)\left(\mu ; a_{1}, \cdots, a_{n}\right) \\
& =\sum_{j=1}^{k} h_{j}{ }^{*}\left(a_{\lambda}\left(\xi_{i}\right)\right) \otimes \sigma\left(h_{j}\right)\left(\mu ; a_{1}, \cdots, a_{n}\right) \\
& =\sigma\left(\sum_{j=1}^{n} h_{j}{ }^{*}\left(a_{\lambda}\left(\xi_{i}\right)\right) h_{j}\right)\left(\mu ; a_{1}, \cdots, a_{n}\right) \\
& =\sigma \circ a_{\lambda}\left(\xi_{i}\right)\left(\mu ; a_{1}, \cdots, a_{n}\right) .
\end{aligned}
$$

Therefore, $\alpha_{\phi_{*} \eta_{1}}=\sigma \circ a_{\lambda}$. The uniqueness of the homomorphism $\phi: C_{1} \longrightarrow \mathcal{R}$ follows from the definition of $\phi$ and the fact that two infinitesimal deformations $\theta$ and $\theta^{\prime}$ are equivalent if, and only if, the corresponding maps $a_{\theta}$ and $a_{\theta^{\prime}}$ are equal, (cf. Theorem 3.10).

\section{Deformation Extensions}

Let us recall some definitions and results from $[\mathbf{1 1}]$. Let $\mathcal{R}$ be a commutative algebra over k. Let $\left(C_{q}(\mathcal{R}), \delta\right)$ denote the standard Hochschild complex, where $C_{q}(\mathcal{R})$ is the $\mathcal{R}$-module $\mathcal{R}^{\otimes(q+1)}$ with $\mathcal{R}$ acting on the first factor by multiplication of $\mathcal{R}$. Let $S h_{q}(\mathcal{R})$ be the $\mathcal{R}$-submodule of $C_{q}(\mathcal{R})$ generated by chains

$$
s_{p}\left(r_{0}, r_{1}, r_{2}, \cdots, r_{q}\right)=\sum_{\sigma \in S h(p, q-p)} \operatorname{sgn}(\sigma)\left(r_{0}, r_{\sigma^{-1}(1)}, r_{\sigma^{-1}(2)}, \cdots, r_{\sigma^{-1}(q)}\right) \in C_{q}(\mathcal{R})
$$

for $r_{1}, r_{2}, \cdots, r_{q} \in \mathcal{R} ; 0<p<q$.

Then $S h_{*}$ is a subcomplex of $C_{*}(\mathcal{R})$, and hence we have a complex called the 
Harrison complex

$$
C h_{*}(\mathcal{R})=\left\{C h_{q}(\mathcal{R}), \delta\right\} ; \quad C h_{q}(\mathcal{R})=C_{q}(\mathcal{R}) / S h_{q}(\mathcal{R}) .
$$

For an $\mathcal{R}$-module $M$, the Harrison cochain complex defining the Harrison cohomology with coefficients in $M$ is given by $C h^{*}(\mathcal{R} ; M)=\operatorname{Hom}_{\mathcal{R}}\left(C h_{*}(\mathcal{R}), M\right)$.

Definition 5.1. For an $\mathcal{R}$-module $M$, we define

$$
H_{\text {Harr }}^{q}(\mathcal{R} ; M)=Z^{q}(\mathcal{R}, M) / B^{q}(\mathcal{R}, M)=H^{q}\left(C h^{*}(\mathcal{R}, M)\right),
$$

where $Z^{q}(\mathcal{R}, M)$ and $B^{q}(\mathcal{R}, M)$ are the spaces of $q$-cocycles and $q$-coboundaries, respectively.

Proposition 5.2. Let $\mathcal{R}$ be a commutative local algebra with maximal ideal $\mathfrak{M}$, and let $M$ be an $\mathcal{R}$-module with $\mathfrak{M} M=0$. Then we have the canonical isomorphism

$$
H_{\text {Harr }}^{q}(\mathcal{R} ; M) \cong H_{\text {Harr }}^{q}(\mathcal{R} ; \mathbf{k}) \otimes M .
$$

Definition 5.3. A (split) abelian extension or square-zero extension $\mathcal{R}^{\prime}$ of $\mathcal{R}$ by an $\mathcal{R}$-module $M$ is a k-algebra $\mathcal{R}^{\prime}$ together with an exact sequence of $\mathbf{k}$-modules

$$
0 \longrightarrow M \stackrel{i}{\longrightarrow} \mathcal{R}^{\prime} \stackrel{p}{\longrightarrow} \mathcal{R} \longrightarrow 0
$$

where $p$ is an algebra homomorphism so that $N=i(M)$ is an $\mathcal{R}^{\prime}$-module and this $\mathcal{R}^{\prime}$ module structure is induced by the $\mathcal{R}$-module structure on $M$ as $r^{\prime} i(m)=i\left(p\left(r^{\prime}\right) m\right)$. In particular, $N$ is an ideal in $\mathcal{R}^{\prime}$ satisfying $N^{2}=0$.

Definition 5.4. An abelian extension $\mathcal{R}^{\prime}$ of $\mathcal{R}$ by $\mathcal{R}$-module $M$ is called a local abelian extension if, in addition, $\mathfrak{M} M=0$, where $\mathfrak{M}$ is the maximal ideal of $\mathcal{R}$.

Henceforth, by an extension we shall always mean a local abelian extension.

Remark 5.5. Note that, as $\mathcal{R}$ is local, $\mathcal{R}^{\prime}$ is also local with $\mathfrak{M}_{\mathcal{R}^{\prime}}=p^{-1}(\mathfrak{M})$ as its maximal ideal. Moreover, the condition $\mathfrak{M} M=0$ clearly implies that, for any $x \in$ $\mathfrak{M}_{\mathcal{R}^{\prime}}$ and $n \in N, x n=0$.

We will use the following results relating Harrison cohomology and extensions of the algebra $\mathcal{R}$ by means of $M,[\mathbf{1 1}]$.

Proposition 5.6. (i) The space $H_{\text {Harr }}^{1}(\mathcal{R} ; M)$ is isomorphic to the space of derivations $\mathcal{R} \longrightarrow M$.

(ii) Elements of $H_{\text {Harr }}^{2}(\mathcal{R} ; M)$ correspond bijectively to isomorphism classes of extensions

$$
0 \longrightarrow M \longrightarrow \mathcal{R}^{\prime} \longrightarrow \mathcal{R} \longrightarrow 0
$$

of the algebra $\mathcal{R}$ by means of $M$.

(iii) The space $H_{\text {Harr }}^{1}(\mathcal{R} ; M)$ can also be interpreted as the group of automorphisms of any given extension of $\mathcal{R}$ by $M$.

Corollary 5.7. If $\mathcal{R}$ is a local algebra with the maximal ideal $\mathfrak{M}$, then

$$
H_{H a r r}^{1}(\mathcal{R} ; \mathbf{k}) \cong\left(\frac{\mathfrak{M}}{\mathfrak{M}^{2}}\right)^{\prime}=T \mathcal{R} .
$$


Let $\mathcal{R}$ be a finite-dimensional commutative, unital, local algebra with augmentation $\epsilon$ and maximal ideal $\mathfrak{M}$. Let $\lambda$ be a deformation of a $\mathcal{P}=\mathcal{P}(\mathbf{k}, E, R)$-algebra $(A, \pi)$ with the base $(\mathcal{R}, \mathfrak{M})$. Let $\left(\mathcal{R}^{\prime}, \mathfrak{M}_{\mathcal{R}^{\prime}}\right)$ be an extension of $(\mathcal{R}, \mathfrak{M})$ by an $\mathcal{R}$-module $M$, where $\operatorname{dim}_{\mathcal{R}} M<\infty$. In this section we consider the problem of extending the given deformation $\lambda$ to a deformation with base $\left(\mathcal{R}^{\prime}, \mathfrak{M}_{\mathcal{R}^{\prime}}\right)$.

First, let us consider the case of a one-dimensional extension. Let

$$
0 \longrightarrow \mathbf{k} \stackrel{i}{\longrightarrow} \mathcal{R}^{\prime} \stackrel{p}{\longrightarrow} \mathcal{R} \longrightarrow 0
$$

be any one-dimensional extension of $\mathcal{R}$. By the above proposition the isomorphism classes of one-dimensional extensions of $\mathcal{R}$ are in one-to-one correspondence with the Harrison cohomology $H_{\text {Harr }}^{2}(\mathcal{R} ; \mathbf{k})$ of $\mathcal{R}$ with coefficients in $\mathbf{k}$ where the $\mathcal{R}$ module structure on $\mathbf{k}$ is given by $r k=\epsilon(r) k$.

Let $[f] \in H_{\text {Harr }}^{2}(\mathcal{R} ; \mathbf{k})$. Suppose $0 \longrightarrow \mathbf{k} \stackrel{i}{\longrightarrow} \mathcal{R}^{\prime} \stackrel{p}{\longrightarrow} \mathcal{R} \longrightarrow 0$ is a representative of the class of one-dimensional extensions of $\mathcal{R}$, corresponding to the cohomology class $[f]$.

Let us recall how the algebra structure on $\mathcal{R}^{\prime}$ is related to $f$. Fix a splitting $q: \mathcal{R} \rightarrow \mathcal{R}^{\prime}$. Let $\hat{\epsilon}=\epsilon \circ p: \mathcal{R}^{\prime} \rightarrow \mathbf{k}$ denote the augmentation of $\mathcal{R}^{\prime}$. Then the map $b \mapsto\left(p(b), i^{-1}(b-q \circ p(b))\right)$ is a k-module isomorphism $\mathcal{R}^{\prime} \cong \mathcal{R} \oplus \mathbf{k}$. Let $(r, k)_{q} \in \mathcal{R}^{\prime}$ denote the inverse of $(r, k) \in \mathcal{R} \oplus \mathbf{k}$ under the above isomorphism. The cocycle $f$ representing the extension is determined by $f\left(r_{1}, r_{2}\right)=i^{-1}\left(\left(r_{1}, 0\right)_{q}\left(r_{2}, 0\right)_{q}-\left(r_{1} r_{2}, 0\right)_{q}\right)$. On the other hand, $f$ determines the algebra structure on $\mathcal{R}^{\prime}$ by

$$
\left(r_{1}, k_{1}\right)_{q} \cdot\left(r_{2}, k_{2}\right)_{q}:=\left(r_{1} r_{2}, r_{1} \cdot k_{2}+r_{2} \cdot k_{1}+f\left(r_{1}, r_{2}\right)\right)_{q} .
$$

As in Section 3, let $\left\{m_{i}\right\}_{i=1}^{r}$ be a fixed basis of the maximal ideal $\mathfrak{M}$ of $\mathcal{R}$ with the dual basis $\left\{\xi_{i}\right\}_{i=1}^{r}$. Let $\psi_{i}=\alpha_{\lambda, \xi_{i}}, 1 \leqslant i \leqslant r$ be the 1 -cochain introduced as in Section 3 . Then by Proposition 3.2, the deformation $\lambda$ can be written as

$$
\lambda=\pi+\sum_{i=1}^{r} m_{i} \otimes \psi_{i},
$$

where $\sum_{i=1}^{r} m_{i} \otimes \psi_{i}$ is a Maurer-Cartan element in $\mathfrak{M} \otimes \mathfrak{g}^{\pi}$.

Let $\left\{n_{j}\right\}_{1 \leqslant j \leqslant r+1}$ be defined by $n_{j}=\left(m_{j}, 0\right)_{q}$ for $1 \leqslant j \leqslant r$ and $n_{r+1}=(0,1)_{q}$. Then $\left\{n_{j}\right\}_{1 \leqslant j \leqslant r+1}$ is a basis of the maximal ideal $\mathfrak{M}_{\mathcal{R}^{\prime}}$ of $\mathcal{R}^{\prime}$.

A deformation $\Gamma$ with base $\mathcal{R}^{\prime}$ extending $\lambda$ is entirely determined by the following two facts:

- $\Gamma$ defined on $\mathcal{P i}^{(1)}$ can be extended to the category of $\mathcal{R}^{\prime}$-modules, and

- if $\hat{\Gamma}$ is the unique extension of $\Gamma$, then $\hat{\Gamma}(r)=0$ for every $r \in R$.

Let $\psi \in \mathfrak{g}^{1}$ be any cochain. Define

$$
\Gamma\left(1_{\mathcal{R}^{\prime}} \otimes \mu\right)=\pi(\mu)+\sum_{j=1}^{r} n_{j} \otimes \psi_{j}(\mu)+n_{r+1} \otimes \psi(\mu),
$$

where $\mu \in \mathcal{P}^{\mathrm{i}^{(1)}}$.

Extending to the category of $\mathcal{R}^{\prime}$-modules, this defines a $\mathcal{R}^{\prime}$-linear map

$$
\Gamma: \mathcal{R}^{\prime} \otimes \mathcal{P}^{(1)} \longrightarrow \mathcal{R}^{\prime} \otimes \operatorname{End}_{A} .
$$

By the universal property of free operads, we extend $\Gamma$ to a morphism of operads 
$\widetilde{\Gamma}: \mathcal{F}\left(E_{\mathcal{R}^{\prime}}\right) \longrightarrow \operatorname{End}\left(A_{\mathcal{R}^{\prime}}\right)$. Now, $\Gamma$ induces a $\mathcal{P}_{\mathcal{R}^{\prime}}$-algebra structure on $A_{\mathcal{R}^{\prime}}$ if, and only if, $\widetilde{\Gamma}((R))=0$, and it is clear from our construction of $\Gamma$ that it extends the given deformation $\lambda$.

As $\lambda$ defines an algebra structure on $\mathcal{R} \otimes A,[\lambda, \lambda]=0$. This implies [13, Proposition 12.2.6])

$$
\sum_{i=1}^{r} m_{i} \otimes \partial_{\pi}\left(\psi_{i}\right)+1 / 2 \sum_{i, j=1}^{r} m_{i} m_{j} \otimes\left[\psi_{i}, \psi_{j}\right]=0 .
$$

Note that saying that $\Gamma$ induces an algebra structure on $\mathcal{R}^{\prime} \otimes A$ is equivalent to saying that, as an element of the Lie algebra $\mathfrak{g}_{\mathcal{R}^{\prime} \otimes \mathcal{P}, \mathcal{R}^{\prime} \otimes A} \cong \mathcal{R}^{\prime} \otimes \mathfrak{g}_{\mathcal{P}, A},[\Gamma, \Gamma]$ vanishes. From the expression of $\Gamma$, we get

$$
\begin{aligned}
{[\Gamma, \Gamma]=} & {\left[\pi+\sum_{i=1}^{r} n_{i} \otimes \psi_{i}+n_{r+1} \otimes \psi, \pi+\sum_{i=1}^{r} n_{i} \otimes \psi_{i}+n_{r+1} \otimes \psi\right] } \\
= & {[\pi, \pi]+2 \sum_{i=1}^{r} n_{i} \otimes \partial_{\pi} \psi_{i}+2 n_{r+1} \otimes \partial_{\pi} \psi+\sum_{i, j=1}^{r} n_{i} n_{j} \otimes\left[\psi_{i}, \psi_{j}\right] } \\
& +2 \sum_{i=1}^{r} n_{i} n_{r+1} \otimes\left[\psi_{i}, \psi\right]+n_{r+1}^{2} \otimes[\psi, \psi],
\end{aligned}
$$

using the fact that $\partial_{\pi}(f)=[\pi, f]$.

We note that $[\pi, \pi]=0$. As $n_{i} n_{r+1}=0$ for $1 \leqslant i \leqslant r(\mathfrak{M k}=0)$ and $n_{r+1}^{2}=0$, the above expression is equal to

$$
2 \sum_{i=1}^{r} n_{i} \otimes \partial_{\pi} \psi_{i}+2 n_{r+1} \otimes \partial_{\pi} \psi+\sum_{i, j=1}^{r} n_{i} n_{j} \otimes\left[\psi_{i}, \psi_{j}\right] .
$$

Thus $\Gamma$ defines a $\mathcal{R}^{\prime} \otimes \mathcal{P}$-algebra structure on $\mathcal{R}^{\prime} \otimes A$ extending $\lambda$, the $\mathcal{R} \otimes \mathcal{P}$ algebra structure on $\mathcal{R} \otimes A$, if, and only if,

$$
\begin{aligned}
& {[\Gamma, \Gamma]=0} \\
& \Longleftrightarrow \quad n_{r+1} \otimes \partial_{\pi} \psi=-\sum_{i=1}^{r} n_{i} \otimes \partial_{\pi} \psi_{i}-\frac{1}{2} \sum_{i, j=1}^{r} n_{i} n_{j} \otimes\left[\psi_{i}, \psi_{j}\right] \\
& \Longleftrightarrow(0,1)_{q} \otimes \partial_{\pi}(\psi)=-\sum_{i=1}^{r}\left(m_{i}, 0\right)_{q} \otimes \partial_{\pi}\left(\psi_{i}\right)-\frac{1}{2} \sum_{i, j=1}^{r}\left(m_{i} m_{j}, f\left(m_{i}, m_{j}\right)\right)_{q} \otimes\left[\psi_{i}, \psi_{j}\right] \\
& \text { (using the isomorphism between } \mathcal{R}^{\prime} \cong \mathcal{R} \oplus \mathbf{k} \text { ) } \\
& \Longleftrightarrow \quad \partial_{\pi}(\psi)=-\frac{1}{2} \sum_{i, j=1}^{r} f\left(m_{i}, m_{j}\right)\left[\psi_{i}, \psi_{j}\right] .
\end{aligned}
$$

Let us define a 2-cochain $\Phi$ on $\mathfrak{g}_{\mathcal{P}, A}$ as follows:

$$
\Phi=\sum_{i, j=1}^{r} f\left(m_{i}, m_{j}\right)\left[\psi_{i}, \psi_{j}\right] .
$$

This cochain is called the obstruction cochain.

Proposition 5.8. The obstruction cochain $\Phi$ is a 2-cocycle in $\mathfrak{g}_{\mathcal{P}, A}$. 
Proof. From equation 5.2,

$$
\sum_{i=1}^{r}\left(m_{i}, 0\right)_{q} \otimes \partial_{\pi} \psi_{i}+1 / 2 \sum_{i, j=1}^{r}\left(m_{i} m_{j}, 0\right)_{q} \otimes\left[\psi_{i}, \psi_{j}\right]=0
$$

as an element of $\mathcal{R}^{\prime} \otimes \mathfrak{g}^{\pi}$.

Let $\partial_{\pi}^{\prime}$ be the differential of $\mathcal{R}^{\prime} \otimes \mathfrak{g}$ induced by $\partial_{\pi}$ in $\mathfrak{g}^{\pi}$. Then

$$
\begin{gathered}
\partial_{\pi}^{\prime}\left(\sum_{i, j=1}^{r} n_{i} n_{j} \otimes\left[\psi_{i}, \psi_{j}\right]\right) \\
=\sum_{i, j=1}^{r} n_{i} n_{j} \otimes \partial_{\pi}\left[\psi_{i}, \psi_{j}\right] \\
=\left(\sum_{i, j=1}^{r} n_{i} n_{j} \otimes\left(\left[\partial_{\pi} \psi_{i}, \psi_{j}\right]-\left[\psi_{i}, \partial_{\pi} \psi_{j}\right]\right)\right) \\
=\left(\left[\sum_{i=1}^{r} n_{i} \otimes \partial_{\pi} \psi_{i}, \sum_{j=1}^{r} n_{j} \otimes \psi_{j}\right]-\left[\sum_{i=1}^{r} n_{i} \otimes \psi_{i}, \sum_{j=1}^{r} n_{j} \otimes \partial_{\pi} \psi_{j}\right]\right) \\
=\left[\sum_{i=1}^{r}\left(m_{i}, 0\right)_{q} \otimes \partial_{\pi} \psi_{i}, \sum_{j=1}^{r}\left(m_{j}, 0\right)_{q} \otimes \psi_{j}\right] \\
=-\frac{1}{2}\left[\sum_{k, l=1}^{r}\left(m_{k} m_{l}, 0\right)_{q} \otimes\left[\psi_{k}, \psi_{l}\right], \sum_{j=1}^{r}\left(m_{j}, 0\right)_{q} \otimes \psi_{j}\right] \\
\left.=\frac{1}{2} \sum_{i, j, k=1}^{r} n_{i}\left(m_{i}, 0\right)_{q} \otimes \psi_{i}, \sum_{j=1}^{r}\left(m_{j}, 0\right)_{q} \otimes \partial_{\pi} \psi_{j}\right] \\
\left.=0, \sum_{i=1}^{r}\left(m_{i}, 0\right)_{q} \otimes \psi_{i}, \sum_{k, l=1}^{r}\left(m_{k} m_{l}, 0\right)_{q} \otimes\left[\psi_{k}, \psi_{l}\right]\right], \text { by }(5.3) \\
=-\frac{1}{2} \sum_{i, j, k=1}^{r}\left(m_{i} m_{j} m_{k}, f\left(m_{i} m_{j}, m_{k}\right)\right)_{q} \otimes\left[\left[\psi_{i}, \psi_{j}\right], \psi_{k}\right] \\
+\frac{1}{2} \sum_{i, j, k=1}^{r}\left(m_{i} m_{j} m_{j} m_{k}, f\left(m_{i}, m_{j} n_{k} \otimes\left(\left[\psi_{i},\left[\psi_{j}, \psi_{k}\right],\right)_{q} \otimes\left[\psi_{i},\left[\psi_{j}, \psi_{k}\right]\right]\right]\right.\right.
\end{gathered}
$$

On the other hand,

$$
\partial_{\pi}^{\prime}\left(\sum_{i, j=1}^{r} n_{i} n_{j} \otimes\left[\psi_{i}, \psi_{j}\right]\right)=\sum_{i, j=1}^{r}\left(m_{i} m_{j}, f\left(m_{i}, m_{j}\right)\right)_{q} \otimes \partial_{\pi}\left[\psi_{i}, \psi_{j}\right] .
$$


Hence, by the previous argument,

$$
\sum_{i, j=1}^{r}\left(m_{i} m_{j}, f\left(m_{i}, m_{j}\right)\right)_{q} \otimes \partial_{\pi}\left[\psi_{i}, \psi_{j}\right]=0 .
$$

By equation (5.2), $\sum_{i, j=1}^{r} m_{i} m_{j} \otimes \partial_{\pi}\left[\psi_{i}, \psi_{j}\right]=0$. Hence,

$$
\partial_{\pi} \Phi=\sum_{i, j=1}^{r} f\left(m_{i}, m_{j}\right) \partial_{\pi}\left[\psi_{i}, \psi_{j}\right]=0 .
$$

The above proposition enables us to define a map from the set of 2-cocycles $Z^{2}(\mathcal{R}, \mathbf{k})$ to $H_{\mathcal{P}}^{2}(A)$. We shall see that this map passes to cohomology.

Let $f$ and $f_{1}$ be two 2-cocycles in the same cohomology class in $H_{\text {Harr }}^{2}(\mathcal{R} ; \mathbf{k})$; that is, $f-f_{1}=\delta h$ for some 1 -cochain $h \in C h^{1}(\mathcal{R}, \mathbf{k})$. The obstruction cocycle for extending $\lambda$ to an extension corresponding to $f$ is given by $\sum_{i, j} f\left(m_{i}, m_{j}\right)\left[\psi_{i}, \psi_{j}\right]$. The obstruction cocycle corresponding to the cocycle $f_{1}$ is given by $\sum_{i, j} f_{1}\left(m_{i}, m_{j}\right)\left[\psi_{i}, \psi_{j}\right]$.
Now,

$$
\begin{aligned}
\sum_{i, j} f\left(m_{i}, m_{j}\right)\left[\psi_{i}, \psi_{j}\right]-\sum_{i, j} f_{1}\left(m_{i}, m_{j}\right)\left[\psi_{i}, \psi_{j}\right]=\sum_{i, j} \delta h\left(m_{i}, m_{j}\right)\left[\psi_{i}, \psi_{j}\right] \\
=\sum_{i, j}\left(m_{i} h\left(m_{j}\right)-h\left(m_{i} m_{j}\right)+h\left(m_{i}\right) m_{j}\right)\left[\psi_{i}, \psi_{j}\right] \\
=-\sum_{i, j} h\left(m_{i}, m_{j}\right)\left[\psi_{i}, \psi_{j}\right] .
\end{aligned}
$$

The terms $m_{i} h\left(m_{j}\right)$ and $h\left(m_{i}\right) m_{j}$ vanish as $\epsilon(\mathfrak{M})=0$. On the other hand, from equation (5.2),

$$
\sum_{i=1}^{r} m_{i} \otimes \partial_{\pi}\left(\psi_{i}\right)=-1 / 2 \sum_{i, j=1}^{r} m_{i} m_{j} \otimes\left[\psi_{i}, \psi_{j}\right]
$$

Hence

$$
\begin{gathered}
(h \otimes \mathrm{id}) \sum_{i=1}^{r} m_{i} \otimes \partial_{\pi} \psi_{i}=-1 / 2 \sum_{i, j=1}^{r} h\left(m_{i} m_{j}\right)\left[\psi_{i}, \psi_{j}\right] \\
\Rightarrow \quad \partial_{\pi}\left(2 \sum_{i=1}^{r} h\left(m_{i}\right) \psi_{i}\right)=\sum_{i=1}^{r} h\left(m_{i}\right) \partial_{\pi}\left(\psi_{i}\right)=-\sum_{i, j=1}^{r} h\left(m_{i} m_{j}\right)\left[\psi_{i}, \psi_{j}\right] .
\end{gathered}
$$

The above consideration defines a map

$$
\theta_{\lambda}: H_{\text {Harr }}^{2}(\mathcal{R} ; \mathbf{k}) \longrightarrow H_{\mathcal{P}}^{2}(A), \quad \text { by } \theta_{\lambda}([f])=[\Phi],
$$

where $[\Phi]$ is the cohomology class of $\Phi$. The map $\theta_{\lambda}$ is called the obstruction map. The proof of the following proposition is straightforward.

Proposition 5.9. Let $\lambda$ be a deformation of a $\mathcal{P}$-algebra $A$ with base $\mathcal{R}$, and let $\mathcal{R}^{\prime}$ be a one-dimensional extension of $\mathcal{R}$ corresponding to the cohomology class $[f] \in$ $H_{\text {Harr }}^{2}(\mathcal{R} ; \mathbf{k})$. Then $\lambda$ can be extended to a deformation of $A$ with base $\mathcal{R}^{\prime}$ if, and only if, the obstruction $\theta_{\lambda}([f])=0$.

We state the following proposition, the proof of which is similar to the proof of Corollary 5.8 in $[6]$. 
Proposition 5.10. Suppose that for a deformation $\lambda$ of a $\mathcal{P}$-algebra $A$ with base $\mathcal{R}$, the differential $d \lambda: T \mathcal{R} \longrightarrow \mathbb{H}$ is onto. Then the group of automorphisms $\mathcal{A}$ of the extension

$$
0 \longrightarrow \mathbf{k} \stackrel{i}{\longrightarrow} \mathcal{R}^{\prime} \stackrel{p}{\longrightarrow} \mathcal{R} \longrightarrow 0
$$

operates transitively on the set of equivalence classes of deformations $\Gamma$ of $A$ with base $\mathcal{R}^{\prime}$ such that $p_{*} \Gamma=\lambda$. In other words, if $\Gamma$ exists, it is unique up to an isomorphism and an automorphism of this extension.

More generally, for an extension

$$
0 \longrightarrow M \stackrel{i}{\longrightarrow} \mathcal{R}^{\prime} \stackrel{p}{\longrightarrow} \mathcal{R} \longrightarrow 0
$$

with $\operatorname{dim}_{\mathcal{R}} M<\infty$, the above arguments can be generalized.

The obstruction map in this more general situation is defined by

$$
\begin{aligned}
\theta_{\lambda}: H_{\text {Harr }}^{2}(\mathcal{R} ; M) & \longrightarrow M \otimes H_{\mathcal{P}}^{2}(A) \\
{[f] } & \mapsto[\Phi] .
\end{aligned}
$$

Then, as in the case of one-dimensional extension, we have the following.

Proposition 5.11. Let $\lambda$ be a deformation of a $\mathcal{P}$-algebra $A$ with base $(\mathcal{R}, \mathfrak{M})$, and let $M$ be a finite-dimensional $\mathcal{R}$-module with $\mathfrak{M} M=0$. Consider an extension $\mathcal{R}^{\prime}$ of $\mathcal{R}$

$$
0 \longrightarrow M \stackrel{i}{\longrightarrow} \mathcal{R}^{\prime} \stackrel{p}{\longrightarrow} \mathcal{R} \longrightarrow 0
$$

corresponding to some $[f] \in H_{\text {Harr }}^{2}(\mathcal{R} ; M)$. A deformation $\Gamma$ of $A$ with base $\mathcal{R}^{\prime}$ such that $p_{*} \Gamma=\lambda$ exists if, and only if, the obstruction $\theta_{\lambda}([f])=0$. If $d \lambda: T \mathcal{R} \longrightarrow \mathbb{H}$ is onto, then the deformation $\Gamma$, if it exists, is unique up to an isomorphism and an automorphism of the above extension.

We end this section with the following naturality property of the obstruction map, the proof of which is similar to Proposition 5.10 in [6].

Proposition 5.12. Suppose $\mathcal{R}_{1}$ and $\mathcal{R}_{2}$ are finite-dimensional unital local algebras with augmentations $\varepsilon_{1}$ and $\varepsilon_{2}$, respectively. Let $\phi: \mathcal{R}_{2} \longrightarrow \mathcal{R}_{1}$ be an algebra homomorphism with $\phi(1)=1$ and $\varepsilon_{1} \circ \phi=\varepsilon_{2}$. Suppose $\lambda_{2}$ is a deformation of a $\mathcal{P}$-algebra $A$ with base $\mathcal{R}_{2}$ and $\lambda_{1}=\phi_{*} \lambda_{2}$ is the push-out via $\phi$. Then the following diagram commutes:

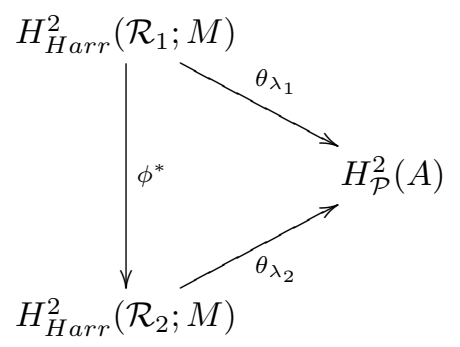

\section{Construction of a Versal Deformation}

We begin the last section with the definition of the notion of a versal deformation of a $\mathcal{P}$-algebra. The importance of versal deformation lies in the fact that it includes 
information of all other non-equivalent deformations of a given object. We use the results developed in the last two sections to give a constructive proof of the existence of versal deformations of a given $\mathcal{P}$-algebra. In fact, starting with the infinitesimal deformation as introduced in Section 4, we give an explicit construction of a versal deformation of a $\mathcal{P}$-algebra by an inductive argument, using the obstruction theory developed in Section 5 .

Definition 6.1. A formal deformation $\eta$ of a $\mathcal{P}$-algebra $A$ with base $\mathcal{R}^{\prime}$ is called versal if it satisfies the conditions

(i) for any formal deformation $\lambda$ of $A$ with base $\mathcal{R}$ there exists a homomorphism $f: \mathcal{R}^{\prime} \longrightarrow \mathcal{R}$ such that the deformation $\lambda$ is equivalent to $f_{*} \eta$;

(ii) if $\mathcal{R}$ satisfies the condition $\mathfrak{M}^{2}=0$, then $f$ is unique.

We proceed to construct a versal deformation of a $\mathcal{P}$-algebra $A$. As before, let $\mathbb{H}=H_{\mathcal{P}}^{1}(A)$, and assume that $\operatorname{dim}(\mathbb{H})<\infty$. Let $\eta_{1}$ be the universal infinitesimal deformation with base $\mathcal{C}_{1}$ as constructed in Section 4 . Suppose for some $k \geqslant 1$ we have constructed a finite-dimensional local algebra $\mathcal{C}_{k}$ and a deformation $\eta_{k}$ of $A$ with base $\mathcal{C}_{k}$. Let

$$
\mu: H_{\text {Harr }}^{2}\left(\mathcal{C}_{k} ; \mathbf{k}\right) \longrightarrow\left(\mathcal{C h}_{2}\left(\mathcal{C}_{k}\right)\right)^{\prime}
$$

be a homomorphism sending a cohomology class to a cocycle representing the class. Let

$$
f_{\mathcal{C}_{k}}: C h_{2}\left(\mathcal{C}_{k}\right) \longrightarrow H_{\text {Harr }}^{2}\left(\mathcal{C}_{k} ; \mathbf{k}\right)^{\prime}
$$

be the dual of $\mu$. By Proposition 5.6 (ii), we have the following extension of $\mathcal{C}_{k}$ :

$$
0 \longrightarrow H_{\text {Harr }}^{2}\left(\mathcal{C}_{k} ; \mathbf{k}\right)^{\prime} \stackrel{\bar{i}_{k+1}}{\longrightarrow} \overline{\mathcal{C}}_{k+1} \stackrel{\bar{p}_{k+1}}{\longrightarrow} \mathcal{C}_{k} \longrightarrow 0 .
$$

The corresponding obstruction $\theta_{\eta_{k}}\left(\left[f_{\mathcal{C}_{k}}\right]\right) \in H_{\text {Harr }}^{2}\left(\mathcal{C}_{k} ; \mathbf{k}\right)^{\prime} \otimes H_{\mathcal{P}}^{2}(A)$ gives a linear map $\omega_{k}: H_{\text {Harr }}^{2}\left(\mathcal{C}_{k} ; \mathbf{k}\right) \longrightarrow H_{\mathcal{P}}^{2}(A)$ with the dual map

$$
\omega_{k}{ }^{\prime}: H_{\mathcal{P}}^{2}(A)^{\prime} \longrightarrow H_{\text {Harr }}^{2}\left(\mathcal{C}_{k} ; \mathbf{k}\right)^{\prime} .
$$

We have an induced extension

$$
0 \longrightarrow \operatorname{coker}\left(\omega_{k}^{\prime}\right) \longrightarrow \overline{\mathcal{C}}_{k+1} /\left(\bar{i}_{k+1} \circ \omega_{k}^{\prime}\left(H_{\mathcal{P}}^{2}(A)^{\prime}\right)\right) \longrightarrow \mathcal{C}_{k} \longrightarrow 0 .
$$

Since $\operatorname{coker}\left(\omega_{k}^{\prime}\right) \cong\left(k e r\left(\omega_{k}\right)\right)^{\prime}$, it yields an extension

$$
0 \longrightarrow\left(k e r\left(\omega_{k}\right)\right)^{\prime} \stackrel{i_{k+1}}{\longrightarrow} \mathcal{C}_{k+1} \stackrel{p_{k+1}}{\longrightarrow} \mathcal{C}_{k} \longrightarrow 0,
$$

where $\mathcal{C}_{k+1}=\overline{\mathcal{C}}_{k+1} /\left(\bar{i}_{k+1} \circ \omega_{k}^{\prime}\left(H_{\mathcal{P}}^{2}(A)^{\prime}\right)\right)$ and $i_{k+1}, p_{k+1}$ are the mappings induced by $\bar{i}_{k+1}$ and $\bar{p}_{k+1}$, respectively. Observe that the algebra $\mathcal{C}_{k+1}$ is also local. Since $\mathcal{C}_{k}$ is finite dimensional, the cohomology group $H_{\text {Harr }}^{2}\left(\mathcal{C}_{k} ; \mathbf{k}\right)$ is also finite dimensional, and hence $\mathcal{C}_{k+1}$ is finite dimensional as well.

Remark 6.2. It follows from Proposition 5.2 that the specific extension (6.1) has the following "universality property." For any $\mathcal{C}_{k}$-module $M$ with $\mathfrak{M} M=0,(6.1)$ admits a unique morphism into an arbitrary extension of $\mathcal{C}_{k}$ :

$$
0 \longrightarrow M \longrightarrow \mathcal{R}^{\prime} \longrightarrow \mathcal{C}_{k} \longrightarrow 0 \text {. }
$$


Proposition 6.3. The deformation $\eta_{k}$ with base $\mathcal{C}_{k}$ of a $\mathcal{P}$-algebra $A$ admits an extension to a deformation with base $\mathcal{C}_{k+1}$, that is unique up to an isomorphism and an automorphism of the extension

$$
0 \longrightarrow\left(k e r\left(\omega_{k}\right)\right)^{\prime} \stackrel{i_{k+1}}{\longrightarrow} \mathcal{C}_{k+1} \stackrel{p_{k+1}}{\longrightarrow} \mathcal{C}_{k} \longrightarrow 0 .
$$

Proof. From the above construction of the extension in 6.2, it is clear that $\theta_{\eta_{k}}\left(\left[f_{\mathcal{C}_{k}}\right]\right)=$ $\left.\omega_{k}\right|_{k e r\left(\omega_{k}\right)}=0$. Therefore, the proof is complete by Proposition 5.11.

By induction, the above process gives a sequence of finite-dimensional local algebras $C_{k}$ and the deformation $\eta_{k}$ of the $\mathcal{P}$-algebra $A$ with base $C_{k}$ :

$$
\mathbf{k} \stackrel{p_{1}}{\longleftarrow} \mathcal{C}_{1} \stackrel{p_{2}}{\longleftarrow} \cdots \stackrel{p_{k}}{\longleftarrow} \mathcal{C}_{k} \stackrel{p_{k+1}}{\longleftarrow} \mathcal{C}_{k+1}
$$

such that $\left(p_{k+1}\right)_{*} \eta_{k+1}=\eta_{k}$.

As a consequence, we obtain a formal deformation $\eta$ of the $\mathcal{P}$-algebra $A$ with base $\mathcal{C}=\lim _{k \rightarrow \infty} \mathcal{C}_{k}$.

The proof of the following theorem is analogous to the proof of Theorem 6.8 of [6].

Theorem 6.4. Let $A$ be a $\mathcal{P}$-algebra with $\operatorname{dim}(\mathbb{H})<\infty$. Then the formal deformation $\eta$ with base $\mathcal{C}$ constructed above is a versal deformation of $A$.

\section{Acknowledgments}

We express our sincere gratitude to the anonymous referee for his valuable comments, which helped us to improve an earlier version of this paper.

\section{References}

[1] D. Balavoine, Deformations of algebras over a quadratic operad, Contemp. Math. 202 (1997), 207-234.

[2] H.-J. Baues, M. Jibladze, and A. Tonks, Cohomology of monoids in monoidal categories, Contemp. Math. 202 (1997), 137-165.

[3] A. Fialowski, Deformations of Lie algebras, Math. USSR-Sbornyik 55 (1986), 467-473.

[4] A. Fialowski and D.B. Fuchs, Construction of versal deformation of Lie algebras, Journal of Funct. Anal. 161 (1999), 76-110.

[5] A. Fialowski and M. Penkava, Deformation Theory of Infinity Algebras, J. Algebra 255 (2002), 59-88.

[6] A. Fialowski, A. Mandal, and G. Mukherjee, Versal deformations of Leibniz Algebras, J. K-Theory 3 (2009), no. 2, 327-358.

[7] M. Gerstenhaber, The cohomology structure of an associative ring, Ann. Math. 78 (1963), 267-288.

[8] M. Gerstenhaber, On the deformation of rings and algebras, Ann. Math. 79 (1964), 59-103.

[9] E. Getzler and J.D.S Jones, Operads, homotopy algebras and iterated integrals for double loop spaces, hep-th/9403055 (1994). 
[10] V. Ginzburg and M.M. Kapranov, Koszul duality for operads, Duke Math. J. 76 (1994), 203-272.

[11] D.K. Harrison, Commutative algebras and cohomology, Trans. Amer. Math. Soc. 104 (1962), 191-204.

[12] M. Kontsevich and Y. Soibelman, Deformations of algebras over operads and the Deligne conjecture, Conférence Moshé Flato 1999, Vol. II (Dijon), Math. Phys. Stud. 22, Kluwer, 2000, 255-307.

[13] J.-L. Loday and B. Vallette, Algebraic operads, Grundlehren der mathematischen Wissenschaften, 346, Springer, 2012.

[14] M. Markl, Models for operads, Comm. Alg. 24 (1996), 1471-1500.

[15] M. Markl, S. Shnider, and J. Stasheff, Operads in Algebra, Topology and Physics, Math. Surveys and Monographs 96, Amer. Math. Soc., 2002.

[16] J.P. May, The geometry of iterated loop spaces, Lectures Notes in Math. 271, Springer-Verlag, 1972.

[17] J.P. May, Definitions: Operads, algebras and modules, in Operads: Proceedings of Renaissance Conferences (Hartford, CT/Luminy, 1995), 1-7, Contemp. Math. 202, Amer. Math. Soc., Providence, RI, 1997.

[18] M. Schlessinger, Functors of Artin Rings, Trans. Amer. Math. Soc. 130 (1968), $208-222$.

Alice Fialowski fialowsk@cs.elte.hu

Institute of Mathematics, Eotvos Lorand University, Pazmany Peter setany 1/C, H1117 Budapest, Hungary

Goutam Mukherjee goutam@isical.ac.in

Stat-Math Unit, Indian Statistical Institute, 203, B. T. Road, Calcutta- 700108, India

Anita Naolekar anita@isibang.ac.in

Stat-Math Unit, Indian Statistical Institute, 8th Mile, Mysore Road, RVCE Post, Bangalore- 560059, India 